\title{
A RAPID AND FACILE DOMINO SYNTHESIS OF STRUCTURALLY DIVERSE 3, 4-DIHYDROPYRIMIDIN-2(1H)- THIONES DERIVATIVES
}

\author{
Suresh Rai ${ }^{1,3, *}$, Anand Kumar Arya ${ }^{2}$, Dilip Kumar Khatri ${ }^{1}$ and Rekha Israni ${ }^{3}$ \\ ${ }^{1}$ Quality Control Department, Panipat Refinery \& Petrochemical Complex, \\ Panipat-132140 (Haryana) India \\ ${ }^{2}$ DESM, Regional Institute of Education (NCERT), Ajmer-305004 (Rajasthan) India \\ ${ }^{3}$ Bhagwant University, Ajmer-305004 (Rajasthan) India \\ *E-mail : rai2iocl@gmail.com
}

\begin{abstract}
An efficient one-pot synthesis of structurally diverse 3,4-dihydropyrimidin-2(1H)-thiones derivatives by $p$-TSA promoted three component reaction of ethyl acetoacetate with thiourea/phenylthiourea and aryl aldehydes is described. The present protocol provides excellent yields of structurally complex, biologically relevant dihydropyrimidin-2(1H)-thiones in a single operation.
\end{abstract}

Keywords: Multicomponent Domino reactions (MDRs), DHPMs, Thiourea, Thiones.

(C) RASĀYAN. All rights reserved

\section{INTRODUCTION}

Multicomponent Domino reactions (MDRs) have emerged as a versatile protocol for the efficient construction of highly functionalized complex molecule in single transformation avoiding the complicated purification procedures, saving both solvents and reagents. ${ }^{1}$ Due to the inherent characteristics of MDRs like atom efficiency, flexibility, ease of execution, diversity orientation and high convergent nature, they are the essential tool for the generation of heterocyclic libraries with high molecular complexity and diversity. ${ }^{2}$ In the recent years, the designing of MCRs for drug discovery has emerged as new endeavor of research. ${ }^{3}$

Nitrogen and sulphur-containing heterocycles are widespread in nature and play a significant role in chemical biology. Among these, 3,4-dihydropyrimidin-2(1H)-ones (DHPMs) and their sulphur analogues are associated with unique biological activities such as antitumor, antihypertensive, anti-HIV, antimicrobials, anti-inflammatory, antifungal, and anthelmintic activities, kinase inhibitors, anticancer, anti-malarial, antihypertensive, potassium channel antagonists, anti-HIV, anti-epileptics, anti-tubercular, anti-bacterial etc. ${ }^{4-14}$ The sulphur derivatives of Dihydropyrimidines are the core structural skeleton in variety of drugs and pharmaceutical potentials compounds ${ }^{15}$ (Figure-1).

The Biginelli-product DHPMs have always been the elegant target of synthesis. Recently, the DHPMs derivatives were reported to be synthesized by solvent-free ball milling technique using ZnO NPs as catalyst. ${ }^{16}$ The 5-phosphonato- and 4-methyl heteroaryl- derivative of DHPMs have been reported respectively as anti-inflammatory ${ }^{17}$ and hepatitis B Virus (HBV) capsid inhibitors. ${ }^{18}$ Encouraged by their recent synthetic protocol and diverse biological and pharmacological importance for drug discovery, we have been interested in developing efficient methodology for the synthesis of 3,4-dihydropyrimidin$2(1 H)$-thiones derivatives.

\section{Material and Methods}

\section{EXPERIMENITAL}

Melting points were measured on the electro thermal melting point apparatus using open capillary tube and were uncorrected. All reagents were commercial grade. The purity of all the synthesized compounds was checked by TLC. IR spectra were measured with a Shimadzu 8400S FTIR spectrometer.

Rasayan J. Chem., 10(2), 471 - 480(2017)

http://dx.doi.org/10.7324/RJC.2017.1021728 
${ }^{1} \mathrm{H}$ NMR and ${ }^{13} \mathrm{C}$ NMR spectra were recorded on Bruker DRX-300 Advance Spectrometer as DMSO-d6. Chemical shifts $(\delta)$ are expressed in ppm downfield from the internal standard tetramethylsilane.

\section{General procedure}

A round bottom flask containing $6 \mathrm{~mL}$ of ethanol was charged with ethyl acetoacetate (1mmol), aryl aldehyde $(1 \mathrm{mmol})$ and substituted thiourea $(1 \mathrm{mmol})$ and $p$-TSA $(10 \mathrm{~mol} \%)$. The reaction mixture was stirred for appropriate time at $80{ }^{\circ} \mathrm{C}$. The progress of the reaction was monitored by TLC. After completion of the reaction (TLC), the solid was filtered off. The solid obtained was washed with distilled water and cold ethanol and purified further by recrystallization from ethanol to yield the pure products.<smiles>CCOC(=O)C1=C(C)NC(=S)N[C@H]1c1cccc(O)c1</smiles><smiles>CC1=C(C(=O)c2ccco2)C(c2cccc(O)c2)NC(=S)N1</smiles><smiles>COc1ccc([C@@H]2NC(=S)N[C@](O)(C(F)(F)F)[C@H]2C(=O)C2CCCO2)cc1</smiles>

(S)-monastrol

1<smiles>[R]NC(=O)C([R])N([R])C(=O)CCCCCN1C(=O)NC([R])C(C(=O)OCc2ccccc2)C1C</smiles>

4
2<smiles>CCOC(=O)C1=C(C(F)(F)F)NC(SCC(=O)c2ccc(C(F)(F)F)cc2)N[C@H]1c1ccc2c(c1)OCO2</smiles>

Fig.-1: Biologically active DHPMs Derivatives

\section{RESULTS AND DISCUSSION}

Initially, to achieve suitable conditions, for the synthesis of 3,4-dihydropyrimidin-2-(1H)-thione a representative Biginelli-type reaction of ethyl acetoacetate $1(1 \mathrm{mmol})$ with phenylthiourea $\mathbf{1}(1 \mathrm{mmol})$ and 3-hydroxybenzaldehyde $\mathbf{3}$ (1 $\mathrm{mmol})$ was performed as a simple model substrate in various solvents in the presence of $\mathrm{FeCl}_{3}, p$-TSA and $\mathrm{CuI}$ as an inexpensive and readily available catalyst (Scheme-1).

To search the suitable solvent, the reaction was examined in various solvents such as methanol, ethanol, DMF and dichloromethane under refluxing condition (Table-1).

As is shown in Table-1, in refluxing organic solvents, ethanol has provided higher yield (Table-1, entry 4). It was observed that the excellent yield of products have obtained when the reaction was performed in the presence of $p$-TSA in ethanol (Table-2, entry 4), while without catalyst trace product was formed after 4 hrs.( Table-2, entry 4). The 10 mol $\%$ of $p$-TSA as catalyst is sufficient to complete the reaction in 24 
min with $93 \%$ yield. The optimal reaction temperature was also explored by performing reaction in ethanol with $p$-TSA at different temperatures range from $60{ }^{\circ} \mathrm{C}$ to $90{ }^{\circ} \mathrm{C}$. The excellent results were obtained at $80^{\circ} \mathrm{C}$.

With the optimized reaction conditions, to explore its scope and generality, the present methodology was extended for library construction (Table-2). The results summarized in Table-2 showed that substituted thiourea and aryl aldehydes react efficiently with ethyl acetoacetate to give the desired product in excellent yields.

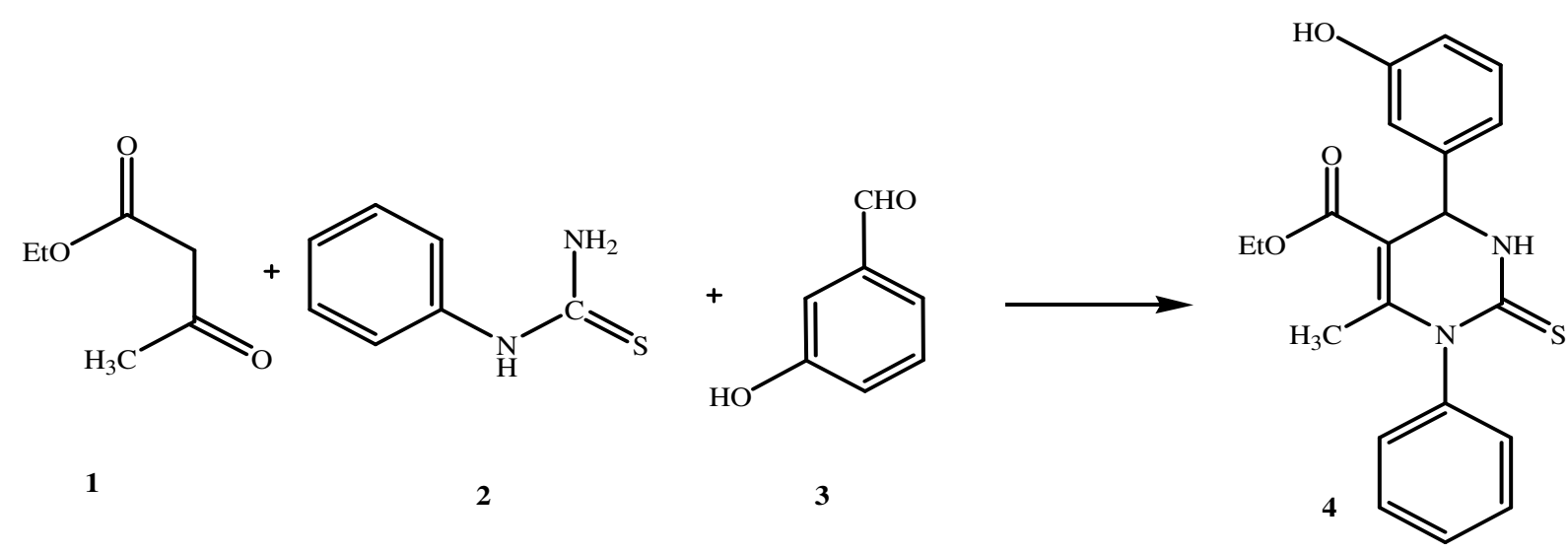

Scheme-1: Model Reaction

Table-1: Model reaction, conditions and yield

\begin{tabular}{l|c|c|c|c}
\hline Entry & Solvent & Catalyst & Time & Yield (\%) \\
\hline 1. & Methanol & $\mathrm{FeCl}_{3}$ & $50 \mathrm{~min}$ & 92 \\
\hline 2. & Methanol & $p$-TSA & $46 \mathrm{~min}$ & 89 \\
\hline 3. & Methanol & $\mathrm{CuI}$ & $1 \mathrm{hrs} 10 \mathrm{~min}$ & 85 \\
\hline 4. & Ethanol & $p$-TSA & $24 \mathrm{~min}$ & 93 \\
\hline 5. & Ethanol & $\mathrm{CuI}$ & $55 \mathrm{~min}$ & 90 \\
\hline 6. & Ethanol & $\mathrm{FeCl}_{3}$ & $60 \mathrm{~min}$ & 85 \\
\hline 7. & Ethanol & No catalyst & more than $4 \mathrm{hrs}$ & traces \\
\hline 8. & DMF & $p$-TSA & $1 \mathrm{hrs} 25 \mathrm{~min}$ & 80 \\
\hline 9. & DMF & $\mathrm{FeCl}_{3}$ & 60 & 82 \\
\hline 10. & Dichloromethane & $p$-TSA & $1 \mathrm{hrs} 40 \mathrm{~min}$ & 76 \\
\hline
\end{tabular}

3-hydroxybenzaldehyde (1 mmol), ethyl acetoacetate $(1 \mathrm{mmol})$, phenylthiourea $(1 \mathrm{mmol})$ and catalyst $(10 \mathrm{~mol} \%)$

5-Ethoxycarbonyl-1-phenyl-4-(3-hydroxyphenyl-)-6-methyl-3,4-dihydropyrimidin-2(1H)-thione (4a) M.p. $139-141^{\circ} \mathrm{C}$, IR (KBr, $\left.v \max , \mathrm{cm}^{-1}\right) ; 3395,3281,1733,1613,1210,1014 .{ }^{1} \mathrm{H}$ NMR (DMSO- $\left.d_{6}\right) \delta$ (ppm): $1.30\left(3 \mathrm{H}, s, \mathrm{CH}_{3}\right), 1.73\left(3 \mathrm{H}, t, \mathrm{CH}_{3}\right), 2.04(1 \mathrm{H}, s, \mathrm{NH}), 4.17\left(2 \mathrm{H}, q, \mathrm{CH}_{2}\right), 4.62(1 \mathrm{H}, s, \mathrm{CH})$, $5.1(1 \mathrm{H}, s, \mathrm{OH}), 6.42-7.06(9 \mathrm{H}, m, \mathrm{H}-\mathrm{Ar}),{ }^{13} \mathrm{C}$ NMR (DMSO- $\left.d_{6}\right) \delta(\mathrm{ppm}): 171.1,165.3,147.1,142.6,139.1$, 128.2, 128.5, 127.3, 126.7, 125.5, 124.2, 102.1, 59.5, 54.6, 15.3, 13.9. Anal. Calcd (\%) for $\mathrm{C}_{20} \mathrm{H}_{20} \mathrm{~N}_{2} \mathrm{O}_{3} \mathrm{~S}$ : C, 65.20; H, 5.47; N, 7.60; found C, 65.14; H, 5.44; N, 7.53.

\section{5-Ethoxycarbonyl-1,4-diphenyl-6-methyl-3,4-dihydropyrimidin-2(1H)-thione $(4 b)$}

M.p. $137-139^{\circ} \mathrm{C}$, IR (KBr, $v$ max, $\left.\mathrm{cm}^{-1}\right)$; 3285, 1742, 1620, 1230, 1020. ${ }^{1} \mathrm{H}$ NMR (DMSO- $\left.d_{6}\right) \delta(\mathrm{ppm})$ : $1.31\left(3 \mathrm{H}, s, \mathrm{CH}_{3}\right), 1.71\left(3 \mathrm{H}, t, \mathrm{CH}_{3}\right), 2.0(1 \mathrm{H}, s, \mathrm{NH}), 4.19\left(2 \mathrm{H}, q, \mathrm{CH}_{2}\right), 4.60(1 \mathrm{H}, s, \mathrm{CH}), 6.41-7.16(10 \mathrm{H}$, $m, \mathrm{H}-\mathrm{Ar}$ ), ${ }^{13} \mathrm{C}$ NMR (DMSO- $\left.d_{6}\right) \delta(\mathrm{ppm}): 171.0,165.1,147.7,142.4,139.5,128.8,128.3,127.1,126.5$, 125.3, 124.4, 102.2, 59.9, 54.9, 15.5, 13.8. Anal.Calcd (\%) for $\mathrm{C}_{20} \mathrm{H}_{20} \mathrm{~N}_{2} \mathrm{O}_{2} \mathrm{~S}: \mathrm{C}, 68.16 ; \mathrm{H}, 5.72 ; \mathrm{N}, 7.95$; found C, 68.10; H, 5.69; N, 7.98. 
RASĀYAN J. Chem.

Vol. 10 | No. 2 |471 - 480 | April - June | 2017

Table-2: Synthesis of 3,4-dihydropyrimidin-2(1H)-thione derivatives

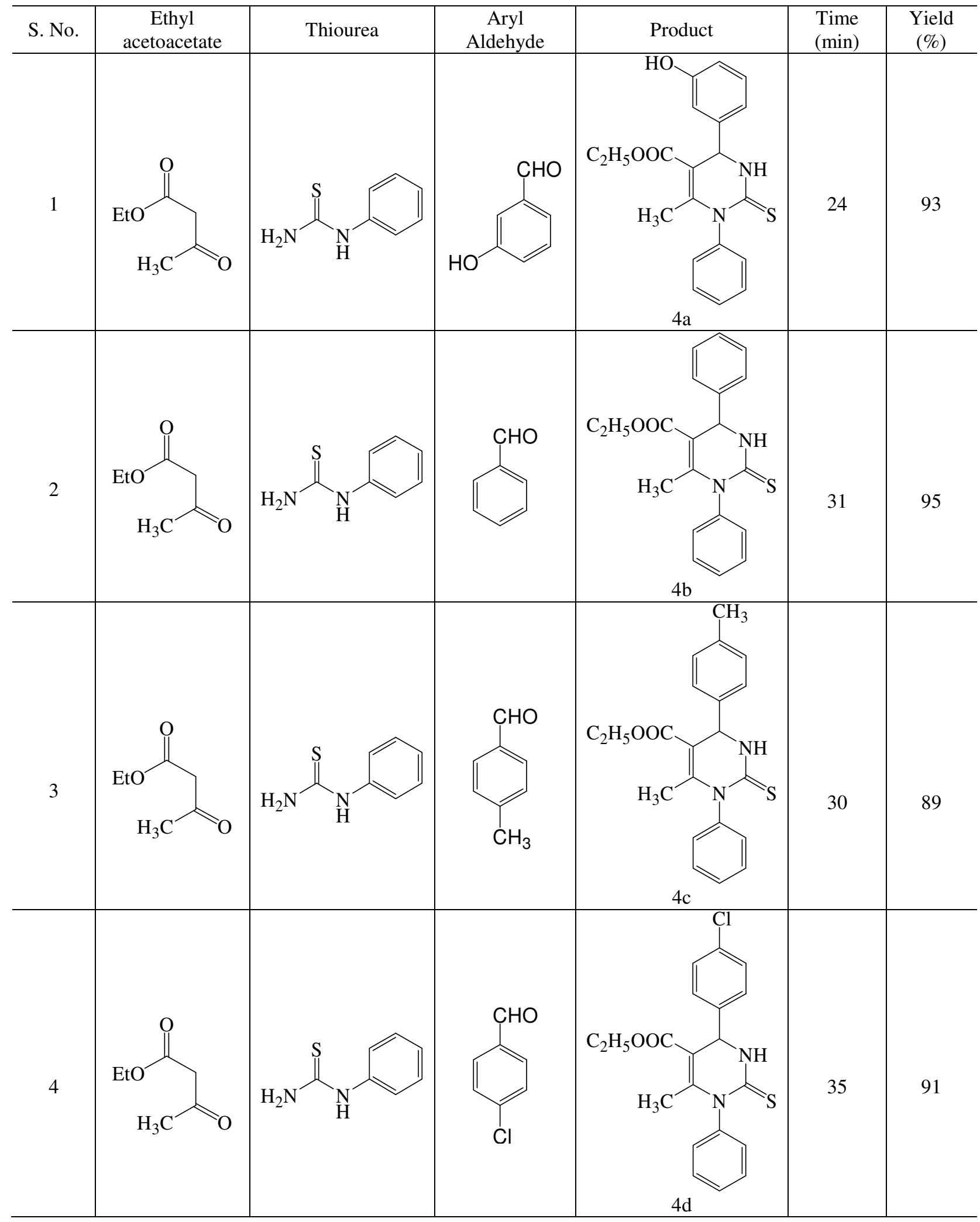


RASĀYAN J. Chem.

Vol. 10 | No. 2 |471 - 480 | April - June | 2017

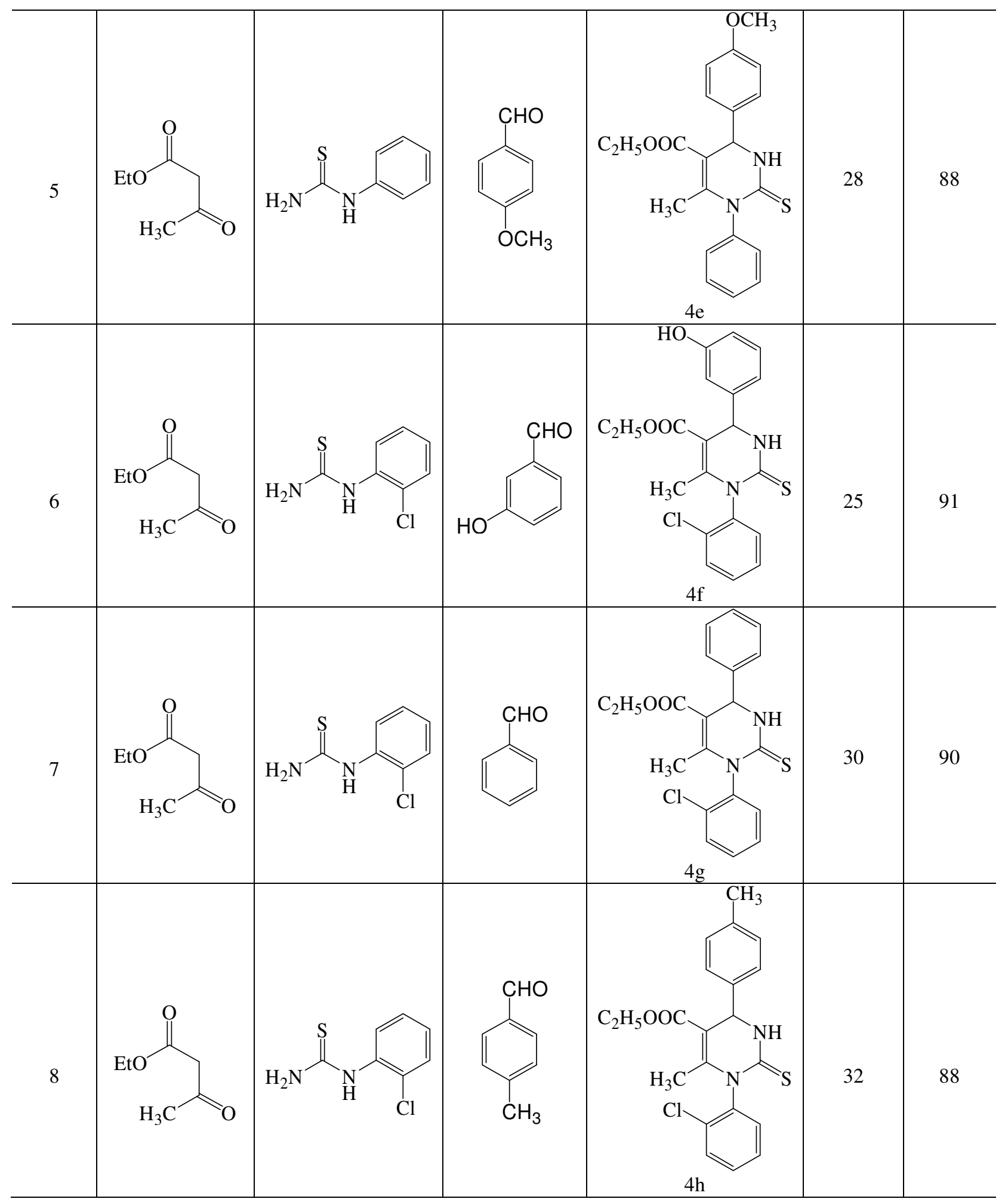


RASĀYAN $J$. Chem.

Vol. 10 | No. 2 |471 - 480 | April - June | 2017

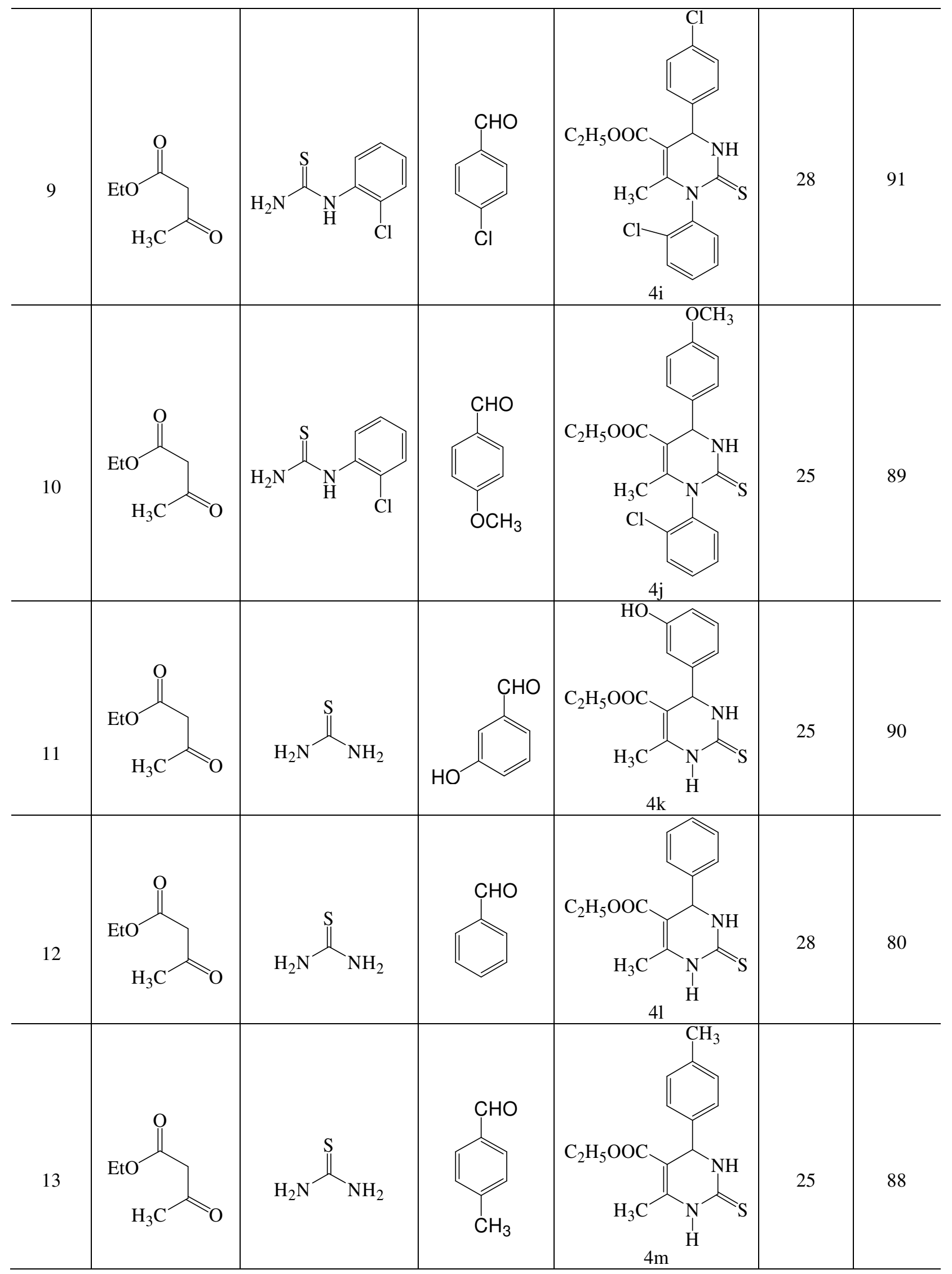




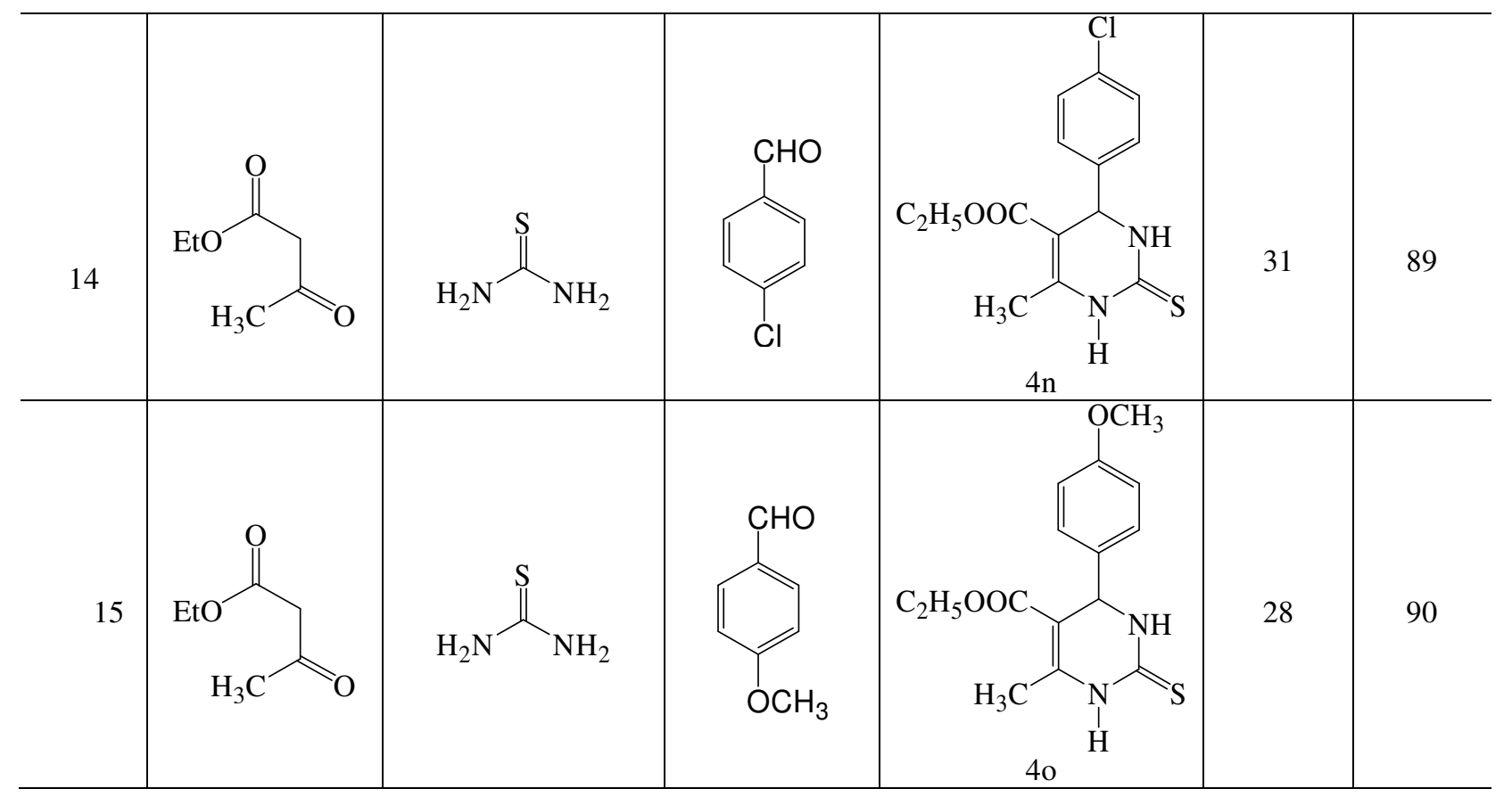

5-Ethoxycarbonyl-6-methyl-4-(4-methoxyphenyl)-1-phenyl-3,4-dihydropyrimidin-2(1H)-thione(4c) M.p.151-153 ${ }^{\circ} \mathrm{C}$, IR $\left(\mathrm{KBr}, v \max , \mathrm{cm}^{-1}\right) ; 3270,1745,1615,1357,1227,1017 .{ }^{1} \mathrm{H}$ NMR (DMSO-d $\left.d_{6}\right)$ $\delta(\mathrm{ppm}): 1.33\left(3 \mathrm{H}, s, \mathrm{CH}_{3}\right), 1.69\left(3 \mathrm{H}, t, \mathrm{CH}_{3}\right), 2.1(1 \mathrm{H}, s, \mathrm{NH}), 2.35\left(3 \mathrm{H}, s, \mathrm{CH}_{3}\right), 4.20\left(2 \mathrm{H}, q, \mathrm{CH}_{2}\right)$, $4.62(1 \mathrm{H}, s, \mathrm{CH}), 6.46-7.01(9 \mathrm{H}, m, \mathrm{H}-\mathrm{Ar}),{ }^{13} \mathrm{C}$ NMR (DMSO- $\left.d_{6}\right) \delta(\mathrm{ppm}): 171.5,165.4,147.9,139.3$, 135.7, 129.1, 128.7, 127.1, 125.2, 124.3, 102.0, 59.7, 54.7, 20.9, 15.4, 13.5. Anal. Calcd (\%) for $\mathrm{C}_{21} \mathrm{H}_{22} \mathrm{~N}_{2} \mathrm{O}_{2} \mathrm{~S}: \mathrm{C}, 68.82 ; \mathrm{H}, 6.05 ; \mathrm{N}, 7.64$; found $\mathrm{C}, 68.75 ; \mathrm{H}, 6.00 ; \mathrm{N}, 7.68$.

5-Ethoxycarbonyl-4-(4-chloroyphenyl)-6-methyl-1-phenyl-3,4-dihydropyrimidin-2(1H)-thione(4d) M.p. $159-161^{\circ} \mathrm{C}$; IR (KBr, $\left.v \max , \mathrm{cm}^{-1}\right) ; 3284,1731,1608,1235,1022 .{ }^{1} \mathrm{H}$ NMR (DMSO- $\left.d_{6}\right) \delta(\mathrm{ppm}): 1.30$ $\left(3 \mathrm{H}, s, \mathrm{CH}_{3}\right), 1.73\left(3 \mathrm{H}, t, \mathrm{CH}_{3}\right), 2.14(1 \mathrm{H}, s, \mathrm{NH}), 4.22\left(2 \mathrm{H}, q, \mathrm{CH}_{2}\right), 4.50(1 \mathrm{H}, s, \mathrm{CH}), 6.42-7.15(9 \mathrm{H}, m, \mathrm{H}-$ Ar), ${ }^{13} \mathrm{C}$ NMR (DMSO- $\left.d_{6}\right) \delta(\mathrm{ppm}): 171.2,165.3,147.5,140.6,139.5,131.8,129.1,128.8,128.7,128.5$, 125.3, 124.7, 102.1, 59.8, 54.8, 15.3, 13.7. Anal. Calcd (\%) for $\mathrm{C}_{20} \mathrm{H}_{19} \mathrm{ClN}_{2} \mathrm{O}_{2} \mathrm{~S}: \mathrm{C}, 62.09 ; \mathrm{H}, 4.95 ; \mathrm{N}$, 7.24; found $\mathrm{C}, 62.01 ; \mathrm{H}, 4.92 ; \mathrm{N}, 7.26$.

5-Ethoxycarbonyl-4-(4-methoxyyphenyl)-6-methyl-1-phenyl-3,4-dihydropyrimidin-2(1H)-thione $(4 e)$ M.p.197-199 ${ }^{\circ} \mathrm{C}$; IR (KBr, $v$ max, $\left.\mathrm{cm}^{-1}\right)$; 3277, 1741, 1625, 1222, 1045, 1017. ${ }^{1} \mathrm{H}$ NMR (DMSO- $d_{6}$ ) $\delta(\mathrm{ppm}): 1.33\left(3 \mathrm{H}, t, \mathrm{CH}_{3}\right), 1.71\left(3 \mathrm{H}, s, \mathrm{CH}_{3}\right), 2.11(1 \mathrm{H}, s, \mathrm{NH}), 3.74\left(3 \mathrm{H}, s, \mathrm{OCH}_{3}\right), 4.20\left(2 \mathrm{H}, q, \mathrm{CH}_{2}\right)$, $4.59(1 \mathrm{H}, s, \mathrm{CH}), 6.46-7.01(9 \mathrm{H}, m, \mathrm{H}-\mathrm{Ar}),{ }^{13} \mathrm{C}$ NMR (DMSO- $\left.d_{6}\right) \delta(\mathrm{ppm}): 171.1,165.5,160.1,147.6$, 139.6, 134.5, 128.8, 128.1, 113.9, 102.2, 59.9, 56.0, 54.9, 15.4, 13.5. Anal. Calcd (\%) for $\mathrm{C}_{21} \mathrm{H}_{22} \mathrm{~N}_{2} \mathrm{O}_{3} \mathrm{~S}$ : C, 65.95; H, 5.80; N, 7.32; found C, 65.89; H, 5.77; N, 7.30.

\section{5-Ethoxycarbonyl-1-(2-chloroyphenyl)-6-methyl-4-(3-hydroxyphenyl)-3,4-dihydro-pyrimidin 2(1H)-thione $(4 f)$}

M.p.155-157 ${ }^{\circ} \mathrm{C}$; IR (KBr, $\left.v \max , \mathrm{cm}^{-1}\right)$; 3381, 3270, 1725, 1632, 1215, 1014. ${ }^{1} \mathrm{H}$ NMR (DMSO- $d_{6}$ ) $\delta(\mathrm{ppm}): 1.33\left(3 \mathrm{H}, t, \mathrm{CH}_{3}\right), 1.68\left(3 \mathrm{H}, s, \mathrm{CH}_{3}\right), 2.2(1 \mathrm{H}, s, \mathrm{NH}), 4.16\left(2 \mathrm{H}, q, \mathrm{CH}_{2}\right), 4.55(1 \mathrm{H}, s$, $\mathrm{CH}), 5.08(1 \mathrm{H}, s, \mathrm{OH}), 6.36-7.18(8 \mathrm{H}, m, \mathrm{H}-\mathrm{Ar}),{ }^{13} \mathrm{C}$ NMR (DMSO- $\left.d_{6}\right) \delta(\mathrm{ppm}): 171.0,165.5,147.2$, $142.7,130.8,129.1,128.5,127.3,126.7,102.5,59.5,56.0,15.4,13.5$. Anal. Calcd (\%) for $\mathrm{C}_{20} \mathrm{H}_{19} \mathrm{ClN}_{2} \mathrm{O}_{3} \mathrm{~S}: \mathrm{C}, 59.62 ; \mathrm{H}, 4.75 ; \mathrm{N}, 6.95 ;$ found $\mathrm{C}, 59.55 ; \mathrm{H}, 4.71 ; \mathrm{N}, 6.94$. 
5-Ethoxycarbonyl-1-(2-chloroyphenyl)-6-methyl-4-phenyl-3,4-dihydropyrimidin-2(1H)-thione(4g) M.p.151-153 ${ }^{\circ} \mathrm{C}$; IR (KBr, $v$ max, $\mathrm{cm}^{-1}$ ); 3280, 1739, 1622, 1235, 1035, 1018. ${ }^{1} \mathrm{H}$ NMR (DMSO- $d_{6}$ ) $\delta(\mathrm{ppm}): 1.30\left(3 \mathrm{H}, t, \mathrm{CH}_{3}\right), 1.71\left(3 \mathrm{H}, s, \mathrm{CH}_{3}\right), 2.1(1 \mathrm{H}, s, \mathrm{NH}), 4.19\left(2 \mathrm{H}, q, \mathrm{CH}_{2}\right), 4.59(1 \mathrm{H}, s, \mathrm{CH}), 6.40-$ 7.14(9H, $m, \mathrm{H}-\mathrm{Ar}),{ }^{13} \mathrm{C}$ NMR (DMSO- $\left.d_{6}\right) \delta(\mathrm{ppm}): 171.5,165.3,147.5,142.4,130.6,129.2,128.3,127.1$, 126.9, 125.9, 102.2, 59.9, 56.0, 54.9, 15.4, 13.7. Anal. Calcd (\%) for $\mathrm{C}_{20} \mathrm{H}_{19} \mathrm{ClN}_{2} \mathrm{O}_{2} \mathrm{~S}$ : C, 62.09; $\mathrm{H}, 4.95$; N, 7.24; found C, 62.00; H, 4.91; N, 7.26.

\section{5-Ethoxycarbonyl-1-(2-chloroyphenyl)-4-(4-methyl-phenyl)-6-methyl-3,4-dihydro-pyrimidin-2(1H)- thione $(\mathbf{4 h})$}

M.p.191-193 ${ }^{\circ} \mathrm{C}$; IR (KBr, $\left.v \max , \mathrm{cm}^{-1}\right)$; 3277, 1747, 1614,1365, 1231, 1038. ${ }^{1} \mathrm{H}$ NMR (DMSO-d $\left.d_{6}\right)$ $\delta(\mathrm{ppm}): 1.29\left(3 \mathrm{H}, t, \mathrm{CH}_{3}\right), 1.73\left(3 \mathrm{H}, s, \mathrm{CH}_{3}\right), 2.0(1 \mathrm{H}, s, \mathrm{NH}), 2.35\left(3 \mathrm{H}, s, \mathrm{CH}_{3}\right), 4.18\left(2 \mathrm{H}, q, \mathrm{CH}_{2}\right)$, $4.55(1 \mathrm{H}, s, \mathrm{CH}), 6.40-7.1(8 \mathrm{H}, m, \mathrm{H}-\mathrm{Ar}),{ }^{13} \mathrm{C}$ NMR (DMSO-d $) \delta(\mathrm{ppm}): 171.3,165.5,147.3,139.8$, 135.6, 130.6, 129.1, 127.0, 126.7,125.9, 102.3, 59.5, 56.2, 54.8, 20.9, 15.2, 13.5. Anal. Calcd (\%) for $\mathrm{C}_{21} \mathrm{H}_{21} \mathrm{ClN}_{2} \mathrm{O}_{2} \mathrm{~S}$ : C, 62.91; H, 5.28; N, 6.99; found C, 62.86; H, 5.22; N, 6.95.

\section{5-Ethoxycarbonyl-1-(2-chloroyphenyl)-4-(4-chlorophenyl)-6-methyl-3,4-dihydropyrimidin-2(1H)- thione (4i)}

M.p.243-245 ${ }^{\circ} \mathrm{C}$; IR (KBr, $v$ max, $\left.\mathrm{cm}^{-1}\right) ; 3287,1731,1624,1223,1023,1017 .{ }^{1} \mathrm{H}$ NMR (DMSO-d $) \delta$ (ppm): $1.33\left(3 \mathrm{H}, t, \mathrm{CH}_{3}\right), 1.71\left(3 \mathrm{H}, s, \mathrm{CH}_{3}\right), 2.10(1 \mathrm{H}, s, \mathrm{NH}), 4.17\left(2 \mathrm{H}, q, \mathrm{CH}_{2}\right), 4.59(1 \mathrm{H}, s, \mathrm{CH}), 6.41-$ 7.16(8H, $m, \mathrm{H}-\mathrm{Ar}),{ }^{13} \mathrm{C}$ NMR (DMSO- $\left.d_{6}\right) \delta(\mathrm{ppm}): 171.5,165.3,147.5,139.5,131.8,128.7,126.6$, 125.7, 102.0, 59.9, 54.2, 15.4, 13.7. Anal.Calcd (\%) for $\mathrm{C}_{20} \mathrm{H}_{18} \mathrm{Cl}_{2} \mathrm{~N}_{2} \mathrm{O}_{2} \mathrm{~S}$ : C, 57.01; H, 4.31; N, 6.65; found $\mathrm{C}, 56.96 ; \mathrm{H}, 4.28 ; \mathrm{N}, 6.61$.

\section{5-Ethoxycarbonyl-1-(2-chloroyphenyl)-4-(4-methoxyphenyl)-6-methyl-3,4-dihydro-pyrimidin- 2(1H)-thione $(4 j)$ \\ M.p. $167-169^{\circ} \mathrm{C}$; IR $\left(\mathrm{KBr}, v \max , \mathrm{cm}^{-1}\right) ; 3271,1729,1607,1234,1045,1011 .{ }^{1} \mathrm{H}$ NMR (DMSO- $\left.d_{6}\right) \delta$ (ppm): $1.31\left(3 \mathrm{H}, t, \mathrm{CH}_{3}\right), 1.69\left(3 \mathrm{H}, s, \mathrm{CH}_{3}\right), 2.15(1 \mathrm{H}, s, \mathrm{NH}), 3.71\left(3 \mathrm{H}, s, \mathrm{OCH}_{3}\right), 4.23\left(2 \mathrm{H}, q, \mathrm{CH}_{2}\right)$, $4.60(1 \mathrm{H}, s, \mathrm{CH}), 6.40-7.02(8 \mathrm{H}, m, \mathrm{H}-\mathrm{Ar}),{ }^{13} \mathrm{C}$ NMR (DMSO-d $) \delta(\mathrm{ppm}): 171.1,165.0,160.3,147.3$, $139.9,137.4,130.8,129.2,128.1,126.2,125.3,113.9,102.2,59.5,54.4,15.1,13.4$. Anal. Calcd (\%) for $\mathrm{C}_{11} \mathrm{H}_{21} \mathrm{ClN}_{2} \mathrm{O}_{3} \mathrm{~S}$ : C, 60.50; H, 5.08; N, 6.72; found C, 60.44; H, 5.00; N, 6.65.}

5-Ethoxycarbonyl-6-methyl-4-(3-hydroxyphenyl)-3,4-dihydropyrimidin-2(1H)-thione (4k)M.p.211$213^{\circ} \mathrm{C}$; IR (KBr, $v$ max $\left.\mathrm{cm}^{-1}\right) ; 3413,3325,3250,1733,1615,1345,1219,1020$. ${ }^{1} \mathrm{H}$ NMR (DMSO- $d_{6}$ ) $\delta(\mathrm{ppm}): 1.26\left(3 \mathrm{H}, t, \mathrm{CH}_{3}\right), 1.71\left(3 \mathrm{H}, s, \mathrm{CH}_{3}\right), 2.01(1 \mathrm{H}, s, \mathrm{NH}), 2.09(1 \mathrm{H}, s, \mathrm{NH}), 4.14\left(2 \mathrm{H}, q, \mathrm{CH}_{2}\right)$, 4.62(1H, $s, \mathrm{CH}), 5.08(1 \mathrm{H}, s, \mathrm{OH}), 7.10-7.15(4 \mathrm{H}, m, \mathrm{H}-\mathrm{Ar}),{ }^{13} \mathrm{C}$ NMR (DMSO- $\left.d_{6}\right) \delta(\mathrm{ppm}): 178.5$, 165.3,152.3,145.2, 128.3,127.1, 126.5, 104.2, 59.9, 54.9, 17.9, 13.7. Anal. Calcd (\%) for $\mathrm{C}_{14} \mathrm{H}_{16} \mathrm{~N}_{2} \mathrm{O}_{3} \mathrm{~S}$ : C, 57.52; H, 5.52; N, 9.58; found C, 57.46; H, 5.50; N, 9.46.

\section{5-Ethoxycarbonyl-6-methyl-4-phenyl-3,4-dihydropyrimidin-2(1H)-thione (4l)}

M.p.207-209 ${ }^{\circ}$ C; IR (KBr, v max, $\left.\mathrm{cm}^{-1}\right)$; 3360, 3280, 1740, 1619, 1365,1229, 1020. ${ }^{1} \mathrm{H}$ NMR (DMSO-d $\left.d_{6}\right)$ $\delta(\mathrm{ppm}): 1.28\left(3 \mathrm{H}, t, \mathrm{CH}_{3}\right), 1.73\left(3 \mathrm{H}, s, \mathrm{CH}_{3}\right), 2.03(1 \mathrm{H}, s, \mathrm{NH}), 2.08(1 \mathrm{H}, s, \mathrm{NH}), 4.17\left(2 \mathrm{H}, q, \mathrm{CH}_{2}\right)$, $4.63(1 \mathrm{H}, s, \mathrm{CH}), 7.06-7.14(5 \mathrm{H}, m, \mathrm{H}-\mathrm{Ar}),{ }^{13} \mathrm{C}$ NMR (DMSO- $\left.d_{6}\right) \delta(\mathrm{ppm}): 178.5,165.3,152.3,145.2$, 128.3, 127.1, 126.5, 104.2, 59.9, 54.8, 17.7, 13.7. Anal. Calcd (\%) for $\mathrm{C}_{14} \mathrm{H}_{16} \mathrm{~N}_{2} \mathrm{O}_{2} \mathrm{~S}: \mathrm{C}, 60.85 ; \mathrm{H}, 5.84$; $\mathrm{N}, 10.14$; found $\mathrm{C}, 60.80 ; \mathrm{H}, 5.80 ; \mathrm{N}, 10.10$.

\section{5-Ethoxycarbonyl-4-(4-methyl-phenyl)-6-methyl-3,4-dihydropyrimidin-2(1H)-thione $(4 m)$}

M.p.149-151 ${ }^{\circ} \mathrm{C}$; IR (KBr, $\left.v \max , \mathrm{cm}^{-1}\right)$; 3354,3279, 1742, 1620, 1230, 1019. ${ }^{1} \mathrm{H}$ NMR (DMSO-d $\left.d_{6}\right)$ $\delta(\mathrm{ppm}): 1.34\left(3 \mathrm{H}, t, \mathrm{CH}_{3}\right), 1.71\left(3 \mathrm{H}, s, \mathrm{CH}_{3}\right), 2.00(1 \mathrm{H}, s, \mathrm{NH}), 2.05(1 \mathrm{H}, s, \mathrm{NH}), 2.34\left(3 \mathrm{H}, s, \mathrm{CH}_{3}\right), 4.22$ $\left(2 \mathrm{H}, q, \mathrm{CH}_{2}\right), 4.58(1 \mathrm{H}, s, \mathrm{CH}), 6.94-6.99(4 \mathrm{H}, m, \mathrm{H}-\mathrm{Ar}),{ }^{13} \mathrm{C}$ NMR (DMSO-d $) \delta(\mathrm{ppm}): 178.5,165.3$, 152.3, 139.4, 135.6, 129.1, 127.0, 104.2, 59.5, 54.6, 17.7, 13.7. Anal. Calcd (\%)for $\mathrm{C}_{15} \mathrm{H}_{18} \mathrm{~N}_{2} \mathrm{O}_{2} \mathrm{~S}: \mathrm{C}$, 62.04; H, 6.25; N, 9.65; found C, 62.00; H, 6.21; N, 9.67. 
RASĀYAN $J$. Chem.

Vol. 10 | No. 2 |471 - 480 | April - June | 2017

5-Ethoxycarbonyl-4-(4-chlorophenyl)-6-methyl-3,4-dihydropyrimidin-2(1H)-thione $(4 n)$

M.p.190-192 ${ }^{\circ} \mathrm{C}$; IR (KBr, $v$ max, $\left.\mathrm{cm}^{-1}\right)$; 3357, 3269, 1739, 1610, 1241,1010. ${ }^{1} \mathrm{H}$ NMR (DMSO- $\left.d_{6}\right) \delta$ (ppm): $1.35\left(3 \mathrm{H}, t, \mathrm{CH}_{3}\right), 1.73\left(3 \mathrm{H}, s, \mathrm{CH}_{3}\right), 2.02(1 \mathrm{H}, s, \mathrm{NH}), 2.08(1 \mathrm{H}, s, \mathrm{NH}), 4.19\left(2 \mathrm{H}, q, \mathrm{CH}_{2}\right)$, $4.55(1 \mathrm{H}, s, \mathrm{CH}), 7.00-7.15(4 \mathrm{H}, m, \mathrm{H}-\mathrm{Ar}),{ }^{13} \mathrm{C}$ NMR (DMSO- $\left.d_{6}\right) \delta(\mathrm{ppm}): 178.3,165.1,152.1,139.6$, 131.8, 128.7, 104.2, 59.6, 54.4, 17.9, 13.9. Anal. Calcd (\%) for $\mathrm{C}_{14} \mathrm{H}_{15} \mathrm{ClN}_{2} \mathrm{O}_{2} \mathrm{~S}: \mathrm{C}, 54.10 ; \mathrm{H}, 4.86 ; \mathrm{N}$, 9.01; found C, 54.06; H, 4.80; N, 9.04 .

\section{5-Ethoxycarbonyl-4-(4-methoxyphenyl)-6-methyl-3,4-dihydropyrimidin-2(1H)-thione (4o)}

M.p. $150-152^{\circ} \mathrm{C}$; IR (KBr, $v$ max $\mathrm{cm}^{-1}$ ); 3345, 3255, 1725, 1608, 1247, 1045, 1019. ${ }^{1} \mathrm{H}$ NMR (DMSO- $d_{6}$ ) $\delta(\mathrm{ppm}): 1.31\left(3 \mathrm{H}, t, \mathrm{CH}_{3}\right), 1.70\left(3 \mathrm{H}, s, \mathrm{CH}_{3}\right), 2.00(1 \mathrm{H}, s, \mathrm{NH}), 2.05(1 \mathrm{H}, s, \mathrm{NH}), 3.73\left(3 \mathrm{H}, s, \mathrm{OCH}_{3}\right), 4.17$ $\left(2 \mathrm{H}, q, \mathrm{CH}_{2}\right), 4.58(1 \mathrm{H}, s, \mathrm{CH}), 6.65-4.95(4 \mathrm{H}, m, \mathrm{H}-\mathrm{Ar}),{ }^{13} \mathrm{C}$ NMR (DMSO- $\left.d_{6}\right) \delta(\mathrm{ppm}): 178.5,165.3$, 160.2, 152.3, 134.7, 128.1, 113.9, 104.2, 59.8, 56.0, 54.6, 17.7, 13.5. Anal. Calcd (\%) for $\mathrm{C}_{15} \mathrm{H}_{18} \mathrm{~N}_{2} \mathrm{O}_{3} \mathrm{~S}$ : C, 58.80; H, 5.92; N, 9.14; found C, 58.75; H, 5.89; N, 9.16.

\section{CONCLUSION}

In conclusion, a rapid and highly efficient approach to structurally diverse 3,4-dihydropyrimidin-2(1H)thiones derivatives has been developed via $p$-TSA-promoted one-pot reaction of ethyl acetoacetate with substituted thiourea/phenyl thiourea and aryl aldehydes in ethanol. The mild conditions, short reaction time, easy purification and easy available starting materials make the protocol interesting for academic research.

\section{REFERENCES}

1. M. Haji, J. Org. Chem., 12, 1269 (2016); (b) K. Ramesh and G. Satyanarayana, J. Org. Chem., 82, 4254 (2017); (c) B. H. Rotstein, S. Zaretsky, V. Rai, A. K. Yudin, Chem. Rev., 114, 8323 (2014).

2. (a) I. A. Wani, M. Sayyad and M. K. Ghorai, Chem. Commun., 53, 4386 (2017); (b) S. Brauch, S. S. van Berkel and B. Westermann, Chem. Soc. Rev., 42, 4948 (2013).

3. (a) A. Dömling, W. Wang and K. Wang, Chem. Rev., 112, 3083 (2012); (b) L. Weber, Curr. Med. Chem., 9, 2085 (2002); (c) B. B. Toure and D. G. Hall, Chem. Rev., 109, 4439 (2009).

4. T. S. Hafez, S. A. Osman, H. A. A. Yosef, A. S. Abd El-All, A. S. Hassan, A. A. El-Sawy, M. M. Abdallah and M. Youns, Sci. Pharm., 81, 339 (2013).

5. (a) O. Alam, S. A. Khan, N. Siddiqui, W. Ahsan, S. P. Verma, S. J. Gilani, Eur. J. Med. Chem., 45, 5113 (2010); (b) C. A. Sehon, G. Z. Wang, A. Q. Viet, K. B. Goodman, S. E. Dowdell, P. A. Elkins, S. F. Semus, C. Evans, L. J. Jolivette, R. B. Kirkpatrick, E. Dul, S. S. Khandekar, T. Yi, L. L. Wright, G. K. Smith, D. J. Behm, R. Bentley, C. P. Doe, E. Hu and D. Lee, J. Med. Chem., 51, 6631 (2008).

6. A. D. Patil, N. V. Kumar, W. C. Kokke, M. F. Bean, A. J. Freyer, C. De Brosse, S. Mai, A. Truneh, B. Carte, J. Org. Chem., 60, 1182 (1995).

7. B. Ramesh and C. M. Bhalgat, Eur J. Med. Chem., 46, 1882 (2011).

8. S. Schenone, M. Radi, F. Musumeci, C. Brullo and M. Botta, Chem. Rev., 114, 7189 (2014).

9. (a) H. Yi K. Kaan, V. Ulaganathan, O. Rath, H. Prokopcová, D. Dallinger, C. O. Kappe and F. Kozielski, J. Med. Chem., 5, 5676 (2010); (b) D. A. Ibrahim and A. M. El- Metwally. Eur. J. Med. Chem., 45, 1158 (2010).

10. A. N. Chiang, J. C. Valderramos, R. Balachandran, R. J. Chovatiya, B. P. Mead, C. Schneider, S. L. Bell, M. G. Klein, D. M. Huryn, X. S. Chen, B. W. Day, D. A. Fidock, P. Wipf, J. L. Brodsky, Bioorg. Med. Chem., 17, 1527 (2009).

11. J. Lloyd, H. J. Finlay, W. Vacarro, T. Hyunh, A. Kover, R. Bhandaru, L. Yan, K. Atwal, M. L. Conder, T. Jenkins-West, H. Shi, C. Huang, D. Li, H. Sun, P. Levesque, Bioorg. Med. Chem. Lett., 20, 1436 (2010).

12. R. W. Lewis, J. Mabry, J. G. Polisar, K. P. Eagen, B. Ganem, G. P. Hess, Biochemistry, 4, 4841 (2010).

13. A. R. Trivedi, V. R Bhuva, B. H. Dholariya, D. K. Dodiya, V. B. Kataria, V. H. Shah Bioorg. Med. Chem., Lett. 20, 6100 (2010). 
RASĀYAN J. Chem.

Vol. 10 | No. 2 |471 - 480 | April - June | 2017

14. (a) S. Chitra, D. Devanathan, K. Pandiarajan. Eur. J. Med. Chem.,45, 367 (2010); (b) M. B. Deshmukh, S. M. Salunkhe, D. R. Patil, P. V. Anbhule, Eur. J. Med. Chem., 44, 2651 (2009).

15. (a) T. M. Kapoor, T. U. Mayer, M. L. Coughlin, T. J. Mitchison, J. Cell Biol., 150, 975 (2000).

16. T. Raj, H. Sharma, Mayank, A. Singh, T. Aree, N. Kaur, N. Singh, and D. O. Jang, ACS Sustainable Chem. Eng., 5, 1468 (2017).

17. I. Essid, K. Lahbib, W. Kaminsky, C. Ben Nasr, S. Touil, J. Mol. Struct., 1142, 130 (2017)

18. Z. Qiu, X. Lin, M. Zhou, W. Liu, W. Zhu, W. Chen, L. Zhang, H. Guo, G. Liu, M. Wu, M. Huang, Z. Jiang, Z. Xu, N. Zhou, S. Qin, H. Ren, S. Qiu, Y. Zhong, Y. Zhang, X. Zhang, L. Wu, F. Shi, Y. Shen, X. Mao, W. Zhou, J. Yang, Z. Wu, G. Yang, A. V. Mayweg, H. C. Shen, and G. Tang, J. Med. Chem., 59, 7651 (2016).

[RJC-1728/2017] 